\title{
The Role of Dissipation in Developing Hydrogels for Functional Tissue Engineering
}

\author{
Dr. Dominique Pioletti \\ Laboratory of Biomechanical Orthopedics, Institute of Bioengineering \\ École Polytechnique Fédérale De Lausanne, Switzerland
}

Dissipation plays an essential role in the mechanical behavior of materials under cyclic loading. In particular for soft materials, an insufficient dissipative capacity is known as the main reason for the low toughness and inability to resist defect growth in hydrogels. In parallel, a new paradigm on the role of dissipative phenomena in soft tissues and biomaterials recently emerged with experimental observations correlating dissipation and mechanobiology. Dissipation may therefore be related to two different but complementary objectives in the development of hydrogels: increase their fatigue resistant properties and induce new mechanobiological features. As example for the development of an hydrogel, we will focus on the clinical situation related to a focal defect in cartilage. In that situation, a fairly stiff and tough hydrogel is required to sustain the particular mechanical behavior of this tissue. As a supplemental constraint, adhesion of the hydrogel to the cartilage is key to avoid its premature delamination. Dissipation also plays a central role for adhesion related process. In this talk, I will present how by carefully controlling the sources of dissipation, hydrogels with enhanced mechanical and mechanobiological properties can be obtained and can be proposed for functional tissue engineering 